\title{
La forme-camp. Pour une généalogie des lieux de transit et d'internement du présent
}

Camp-shaped: For a genealogy of present transit and internment

\section{Federico Rahola}

Traducteur : Sarah Guilmault

\section{OpenEdition Journals}

\section{Édition électronique}

URL : http://journals.openedition.org/conflits/5213

DOI : 10.4000/conflits.5213

ISSN : $1777-5345$

\section{Éditeur :}

CCLS - Centre d'études sur les conflits lilberté et sécurité, L'Harmattan

Édition imprimée

Date de publication : 30 décembre 2007

Pagination : $31-50$

ISBN : 978-2-296-05120-1

ISSN : $1157-996 X$

\section{Référence électronique}

Federico Rahola, «La forme-camp. Pour une généalogie des lieux de transit et d'internement du présent », Cultures \& Conflits [En ligne], 68 | hiver 2007, mis en ligne le 14 avril 2008, consulté le 30 mars 2021. URL : http://journals.openedition.org/conflits/5213 ; DOI : https://doi.org/10.4000/ conflits. 5213 


\section{La forme-camp. Pour une généalogie des lieux de transit et d'internement du présent ${ }^{1}$}

\section{Federico RAHOLA}

Federico Rabola est chercheur en sociologie des processus culturels à l'université de Gênes et rédacteur des revues Conflitti Globali (XBook) et Etnografia (Il Mulino). Il est également l'auteur, entre autres, de Zone definitivamente temporanee. I luoghi dell'umanità in eccesso, Vérone, Ombre Corte, 2004.

« Notre temps - son impérialisme militaire moderne, les ambitions quasi théologiques de ses gouvernants despotiques et totalitaires - est le temps des réfugiés, des exilés, de l'immigration de masse ${ }^{2}$. »

Th récit extraordinaire de l'écrivain palestinien Ghassan Kanafani, Rijal fil Sharns, datant de $1963^{3}$, décrit la tentative tragique de trois jeunes Palestiniens voulant rejoindre le Koweït. A Bassorah, en Irak, les exilés rencontrent un contrebandier qui transporte des « marchandises » dans le container de son camion-citerne. Ils lui demandent, moyennant argent, s’il peut les emmener jusqu'à la frontière. Lorsqu'ils sont presque arrivés à destination, le conducteur s'arrête sous un soleil de plomb pour discuter avec les gardiens. Il oublie son «chargement» (ou peut-être pas), et les Palestiniens meurent asphyxiés sans que personne ait pu entendre leurs cris.

La littérature et le cinéma ${ }^{4}$ sont sans doute les seuls qui réussissent à saisir avec tant de justesse toute la violence qui appartient à ces frontières : une profusion d'espérances, de tensions et de tragédies qui s'accumulent dans ces lieux

1. Cet article a été initialement publié dans Conflitti Globali, nº4/2007. Traduit de l'italien par Sarah Guimault.

2. Said E., "Reflections on Exile", On Exile and Other Essays, Cambridge, Harvard University Press, 2002.

3. Kanafani G., Des hommes dans le soleil, Paris, Sindbad, 1990.

4. Nous pensons au roman de T.C. Boyle, America, ou à l'un des derniers films de Tommy Lee Jones, Trois enterrements. 
de «transit » répondant aux noms de Tijuana, Gaza, Ceuta et Melilla, les Canaries, Lampedusa. L'histoire de Kanafani se reflète dans chacune des fissures qui meurtrissent la superficie du présent, et à travers tous les naufrages sans spectateurs qui en marquent l'histoire. Mais ce récit n'est pas seulement le miroir fidèle et hyper réaliste d'une tragédie frontalière «banale », il est aussi la métaphore de la condition palestinienne en général. Il est en effet facile de voir à travers la tragédie du container la caricature déformée de la Cisjordanie ou de la bande de Gaza et, dans la mort par asphyxie, le destin politique d'un peuple et peut-être d'un futur Etat à souveraineté " concentrée ». Le container peut même devenir la métaphore plus littérale de la condition particulière que partagent tous ceux qui, comme les Palestiniens, sont contraints de trouver dans des lieux provisoires leur propre territoire permanent et leur propre destin définitif. En tant que tel, il peut faire référence de façon plus générale à toute une humanité déplacée, en transit entre les frontières, dont le destin est toujours menacé par la possibilité d'un enfermement dans un camp ${ }^{5}$.

Ces pages s'interrogent sur le sens que peuvent revêtir de tels lieux, sur les sujets destinés à les habiter et sur l'espace politique auxquels nous devons les relier. Dans un certain sens, l'idée est de partir du récit de Kanafani et de l'utiliser comme métaphore de tous les camps qui infestent le présent, en les reconduisant à une "forme » plus générale, à une matrice commune et en tentant d'en écrire l'histoire.

\section{Définitions}

«Le temps des réfugiés, des exilés, de l'immigration de masse » : ces mots empruntés à la citation en exergue de cet article font allusion à la répartition de cette humanité déplacée. Ils sont extraits d'un essai d'Edward Said, palestinien lui aussi, dédié à cette forme particulière de déplacement qu'on appelle exil ${ }^{6}$ sur un ton vaguement élégiaque. Cette citation nous permet de constater que nous avons atteint un point de non-retour pour lequel l'exil, en tant qu'expérience de persécution spécifiquement individuelle, motivée par des facteurs aberrants mais toujours liés à la biographie individuelle parle de quelque chose que nous ne sommes plus. Aujourd'hui, en effet, le statut individuel des sujets déplacés est le plus souvent effacé et reconduit systématiquement à des catégories totalisantes qui, dans le lexique du droit international et humanitaire, correspondent à une poignée de truismes : « internally displaced (déplacés), « asylum seekers» (demandeurs d'asile), «temporary refugees" ou réfugiés de "prima facie » (individus objectivement persécutés dans leur pays de provenance auxquels on accorde l'asile

5. NdR : Centres de rétention, zones d'attente, centres fermés pour demandeurs d'asile, campstampons à la périphérie de l'Union européenne (au Maroc, en Espagne, en Algérie, en Ukraine, à Malte,...), centres « ouverts » d'accueil ou d'hébergement, portail d'immigration, etc.

6. Said E., op. cit. 
temporaire), jusqu'aux migrants, « économiques » ou non, « réguliers » ou non - et ici le lexique semble perdre son caractère procédural ostentatoire puisqu'il a recours au terme plus connotatif d' "illegal aliens » (étrangers en situation irrégulière).

Ce ne sont que des mots, des exercices nominalistes, pourrait-on dire. En réalité, ces définitions finissent par produire ce qu'elles indiquent, comme ce doigt qui pointe vers la lune. Dans ce cas, il s'agit de classifier des sujets dont la présence est le symptôme d'une condition limite, qui échappe à l'artifice nominaliste qui appartient au droit (un individu, une définition, un statut) parce qu'il excède toute définition univoque, tout critère partitif d'appartenance. Pour pouvoir nommer et classifier cet « excès ", la seule solution sera alors d'annuler tous les éléments individuels en les plaçant au sein de catégories abstraites et cumulatives, ou en les réifiant sur des bases nationales et ethniques puis, sur la base de ces classifications, opérer des choix et décider du sort des individus en question. Il faut cependant prendre en compte au moins deux autres considérations, ou plutôt deux autres précisions.

La première concerne le côté arbitraire que supposent de telles distinctions. S’il est vrai que le sort des trois Palestiniens raconté par Kanafani peut rappeler celui de milliers d'autres individus déplacés, réfugiés, demandeurs d'asile ou migrants, il est également vrai que toutes ces définitions peuvent se superposer en une seule et unique biographie, qui pourrait à elle seule faire l'objet d'un roman. Il est possible, après avoir été contraints d'évacuer leurs propres maisons et entrepris une marche forcée vers " nulle part », de réussir à franchir les frontières nationales pour demander asile à un pays tiers (et nous retrouvons l'« asylum seeker », le "demandeur »). Il se peut ensuite, mais là nous pénétrons dans le domaine des probabilités infimes, que la demande d'asile soit acceptée, même si ce n'est que temporairement, parce que rien ne fait obstacle à la reconnaissance (ils se transforment ainsi en réfugiés de prima facie). Il faut dire que, sauf rares exceptions, les milliers de demandes d'asile qui parviennent quotidiennement aux consulats ou aux postes de police des frontières, restent lettre morte et le refus ne se limite pas à une simple réponse négative, technique et conjoncturelle, mais résonne plutôt comme un décret qui marque définitivement l'histoire personnelle du demandeur. En effet, il lui refuse la possibilité de représenter sa demande dans d'autres pays, dans d'autres bureaux et aux autres polices des frontières : un précédent qui entraîne en somme un véritable banissement ${ }^{7}$. Dans tous les cas, et parce qu'il concerne en général des catégories entières de population, l'asile est aujourd'hui conçu comme un instrument temporaire lié à une possible normalisation de la situation de crise qui caractérise la zone de provenance des groupes en question et à la disparition souhaitable (et in fine établie

7. Sur l'abolition substantielle du droit d'asile et sur sa sinistre reconversion en dispositif de fichage et de profiling des demandeurs, mis définitivement au ban dès le premier refus, nous renvoyons à Valluy J., «La nouvelle Europe politique des camps d'exilés », Cultures E Conflits, n57, 2005. 
arbitrairement) des conditions de vie déplorables qui en légitimait l'octroi ${ }^{8}$. A l'expiration de cette protection temporaire, on suppose que les individus retournent spontanément dans leur pays d'origine. Sinon, pèsera sur ces individus un décret d'expulsion théorique subsumant l'existence des ex-« déplacés » puis demandeurs d'asile et enfin réfugiés temporaires sous le signe de la clandestinité - c'est-à-dire dans cet état d'absolue précarité et soumis à un chantage permanent qui les précipite dans la définition extra-juridique des sans-papiers. Ce ne sont pas tant les différences objectives présumées entre des individus partageant une condition générale de déplacés que l'arbitraire des définitions qui efface les histoires personnelles en les fondant dans des catégories cumulatives, et les effets politiques engendrés par ce type de pratiques définitoires.

Le point alors concerne moins les différences «objectives » présumées entre les individus qui partagent une condition générale de déplacement, que l'arbitraire des définitions qui annulent les biographies en les casant dans des catégories cumulatives, et les effets politiques que des pratiques définitoires de cette sorte engendrent.

La seconde considération concerne l'objet même de cet article, parce qu'à chaque définition, à chaque étape de la « carrière morale 9 » des individus en question, correspondra un camp, un centre temporaire plus ou moins équipé. Même dans ce cas, les formules officielles abondent d'euphémismes et d'expédients rhétoriques. Parmi les « jeux de mots » où l'accent est mis sur le caractère administratif de détention, ou encore sur celui de protection et d'accueil, les définitions qui ne cessent d'insister sur son côté temporaire et précaire se succèdent : hébergement d'urgence et temporaire, pour la catégorie la plus répandue et la moins équipée, zone de protection temporaire (ou encore, dans une formule anglaise plus rhétorique, "safe havens " c'est-à-dire "refuge protégé ») pour les déplacés ; «transit processing centres», ou « centres d'identification », pour les demandeurs d'asile; plus banalement « refugees temporary centres» ou « centres d'accueil temporaire » pour les «temporary refugees»; «detention centres ", «centres de permanence temporaire », zones d'attente pour les migrants irréguliers.

En d'autres termes, moins euphémiques, s'il est théoriquement possible qu'un seul individu puisse endosser toutes les définitions susmentionnées dont nous venons de dresser la liste, on observe la constante politique suivante : à chaque définition, aussi arbitraire puisse-t-elle paraitre, correspond un de ces « centres équipés », une de ces zones définitivement temporaires: lieu de transit, comme le container du récit de Kanafani, dont le caractère

8. Sur l'extension du recours à l'asile temporaire, qui redéfinit radicalement en termes de précarité les droits fixés par la Convention de Genève de 1951, on peut consulter : UNHCR, Draft Directive on Temporary Protection in the Event of a mass Influx, Genève, septembre 2006 :

www.unhcr.org/cgi-bin/texis

9. NdT: «carrière morale » est un concept développé par Erving Goffman. 
absolument provisoire entre en collision avec celui de l'inexorabilité péremptoire, accompagnant et marquant les frontières du présent.

\section{Le camp comme forme}

C'est à partir de cette constance, de ce transit permanent par des lieux temporaires, que prend corps l'hypothèse d'une "forme-camp ", d'une matrice commune capable de rendre compte de toutes les phénoménologies, même éloignées les unes des autres, avec lesquelles les camps font irruption dans le présent ${ }^{10}$. Les camps, dans cette perspective, apparaissent avant tout comme étant le seul territoire " possible » où reconduire et confiner l'humanité en mouvement entre les frontières et les appartenances univoques. Il semblerait donc que ce soit l'indéterminabilité du statut des sujets qui donne un «sens » aux camps : lieux "perdus » pour des sujets «perdus ", réponse qui excède pour des sujets qui excèdent. Le fait est qu'une telle réponse est réductrice, et c'est justement parce qu'elle est excessivement formelle, presque mécanique, qu'elle finit ainsi par diminuer ou supprimer complètement la dimension «dynamique » particulière relative aux camps - dimension à laquelle l'idée même de forme voudrait renvoyer. C'est pour cela qu'en plus du statut de celui qui est contraint de transiter par un camp, il faut prendre en considération la « vie » de ces sujets, et donc leur intervention directe sur les lieux, l'irrésistible rébellion qui traverse chaque camp. Mais d'un autre côté, il est aussi nécessaire de rendre compte de la sinistre complémentarité qui soude entre eux les lieux et les sujets, et donc de la dimension "productive » particulière dont sont investis les camps. La façon dont ils pèsent sur les individus déplacés comme une possibilité menaçante, le fait d'en définir matériellement les conditions de vie dans le temps (en termes de précarité) et dans l'espace (en termes de confinement) ne permettent pas de se débarrasser des camps comme de simples lieux «perdus ». Chaque réflexion sur les camps doit alors se mesurer à une série de questions préliminaires : que signifie le fait d'être désignés comme sujets dont le destin est marqué par un camp ? Quel rôle peut jouer un dispositif comme le camp dans la production de cette condition ? A quelle dimension spécifique de frontière et à quel espace politique renvoie-t-il ?

L'idée d'une «forme-camp» suit essentiellement le tracé de ces questions. Elle indique la nécessité de prendre en considération tous les camps qui peuplent le présent, mais aussi la possibilité de récupérer une trame verticale, un parcours historique à partir duquel on peut relire l'« histoire séparée » désignée par ce dispositif extrême et particulier de confinement. Le point de départ, dans ce cas, nous le retrouvons dans les mots d'Hannah Arendt, extraits d'un chapitre des Origines $d u$ totalitarisme et de notes éparses, dans lesquels les camps d'internement sont définis comme des «substituts du territoire national » où sont relégués les individus qui n’y appartiennent pas :

10. Nous reprenons ici certains concepts développés ultérieurement dans : Rahola F., Zone definitivamente temporanee. I luoghi dell'umanità in eccesso, Vérone, Ombre Corte, 2003. 
"Toute tentative, de la part des conférences internationales, d'instituer un statut juridique pour les apatrides a échoué parce qu'aucun statut internationalement garanti ne pouvait substituer le territoire où chasser un étranger indésirable. Toutes les discussions qui ont lieu depuis trente ans autour de ce problème se sont axées sur une seule question : comment peut-on exiler de nouveau un exilé ? Le seul substitut concret à un pays natal inexistant a toujours été les camps d'internement. De fait, ce fut dès les années 1930 le seul “pays” que le monde eut à offrir aux sans-Etat ${ }^{11}$. »

Au travers de ces quelques lignes, Arendt semble vouloir octroyer au camp d'internement le rôle de matrice spécifique («le seul substitut [...] a toujours été les camps d'internement ») à partir de laquelle il s'avère possible de reconstruire un parcours historique global et sui generis - un parcours capable de dire aussi quelque chose sur les manifestations les plus extrêmes, comme les Lager et les goulags, ne s'arrêtant que face à l'absolu des camps d'extermination ${ }^{12}$. C'est justement sur cette « matrice » qu'il faut s'arrêter un moment pour développer l'intuition arendtienne d'un point de vue conceptuel et géographique.

Le fait que les camps constituent un lieu qui, à différents titres, pèse sur les sujets « hors place » apparaît toujours comme un élément décisif. Les migrants, les réfugiés et les déplacés partagent en effet une condition de déplacement qui peut se définir aussi en termes d'action, comme « défection » qui échappe et vide les catégories politiques qui fixent un individu à un seul et même lieu, réclamant matériellement d'autres formes de (non)-appartenance. Il s'agit cependant d'un « excédent » qui devient en même temps un symptôme évident, qualitatif plus que quantitatif, d'un point de non-retour qui met en crise tout ce qui se montre encore comme étant «à sa place ». Une phrase extraite d'un texte de Stephen Castles et Alistair Davidson synthétise efficacement ce qui émerge ici comme l'un des caractères de fond de l'époque actuelle, à savoir l'implosion des formes dans lesquelles est organisée l'appartenance politique aujourd'hui :

«Des millions de personnes sont privées de leurs droits puisqu'ils ne peuvent devenir citoyens du pays dans lequel ils résident. Plus nombreux encore sont ceux qui ont obtenu le statut formel de membre de l'Etat national sans avoir cependant accès à une grande partie des autres droits qui dérivent normalement de cette condition. [...] Il y a de plus en plus de citoyens qui n'“appartiennent pas”, et cette circonstance mine à son tour la base de l'Etat national en tant que lieu central de la démocratie ${ }^{13}$. »

11. Arendt H., Les Origines du totalitarisme, Paris, Le Seuil, 1998.

12 . Arendt H., "Les techniques de la science sociale et l'étude des camps de concentration », in Courtine-Denamy S. (dir.), Auschwitz et Jérusalem, Paris, Deux-Temps Tierce, 1991.

13. Castles S., Davidson A., Citizenship and Migration. Globalization and the Politics of Belonging, Londres, Macmillan, 2000, p. viii. 
Ces mots soulignent la crise globale d'un système inclusif c'est-à-dire d'un rapport dialectique entre inclusion et exclusion où cette dernière est réabsorbée et dépassée à l'intérieur d'une idée de droit, d'Etat et de démocratie. L'hypothèse que nous avançons ici énonce que l'existence des camps constitue l'indice plus immédiat d'une crise, faisant allusion à un espace " autre ", définitivement au-delà des frontières du droit et de l'Etat même.

Une littérature plutôt abondante décrit les camps d'internement en termes d'exception. Giorgio Agamben, par exemple, relisant les événements de la souveraineté moderne en fonction du couple dialectique «biopouvoir» et "vie nue » 14 envisage le camp comme paradigme absolu de la souveraineté, lieu dans lequel le pouvoir et la vie se confrontent, suspendant toute trace de médiation et de droit. Agamben tente de réfléchir sur ce qui se produit comme étant « extérieur » à un système déterminé, et qui peut être compris, "pris dehors » (qui, comme il le rappelle, vient de l'étymologie de excipere ${ }^{15}$ ), seulement en suspendant les instruments ordinaires du droit et en ayant recours aux armes métaphysiques de la souveraineté, en tant qu'instance suprême qui décrète l'état d'exception. Dans une perspective certainement plus proche de l'idée foucaldienne de gouvernementalité, il serait nécessaire de s'arrêter un instant sur le sens des lieux et des pratiques qui définissent et gouvernent des sujets, sans faire nécessairement appel à des logiques transcendantes de souveraineté qui, au contraire, se réalisent, sur un plan tout à fait immanent, à travers les gestes et les effets immédiats. En d'autres termes, il s'agit de prendre en considération un dispositif comme le camp pour sa capacité productive immédiate, c'est-à-dire, «positive », en référence à Michel Foucault ${ }^{16}$. C'est à partir de cette "positivité » spécifique que l'idée de développer une « formecamp » s'avère possible. Le terme «forme » dans ce cas ne devrait pas être entendu dans son acception commune d'un encadrement neutre, privé de signe et de direction. Au contraire, il est nécessaire de répéter le sens essentiellement dialectique du "principe authentique qui concrétise », d'un «facteur étrange d'attraction qui déforme, compromet et confère une couleur spécifique à chaque élément de la totalité » ${ }^{17}$. Cette idée de forme, si elle est associée à la matrice du camp défini par Arendt, permet de recueillir quelque chose qui " produit une différence». A chaque fois qu'elle fait irruption, elle marque un dépassement par rapport à chaque expérience d'exclusion précédente, signalant une différence radicale. En d'autres termes, à chaque fois que nous avons recours au confinement des camps, ces derniers marquent un seuil, une frontière définitivement franchie. Ils déterminent un type d'exclusion qui va audelà de toute forme de marginalité sociale. Ils cessent de dialoguer avec un

14. Voir Agamben G., Homo sacer, Paris, Le Seuil, 1997 ; Agamben G., Etat d'exception, Paris, Le Seuil, 2003.

15. Agamben G., Homo sacer, op. cit.

16. Foucault M., Sécurité, territoire, population, Paris, Gallimard-Seuil, 2004.

17. Zizek S., Tredici volte Lenin. Per sovvertire il fallimento del presente, Milan, Feltrinelli, 2003, p. 32. 
dedans, et probablement avec un dehors. N'étant plus reconductibles à une économie particulière (celle, par exemple, des institutions totales), ils vont audelà de l'exclusion et ils deviennent le signe de quelque chose d'autre, quelque chose qui fait référence à un excès, qui concerne et définit directement l'existence des individus qui « excèdent » le sens d'un lieu.

"Donner forme », "produire une différence », c'est là que réside la dimension spécifique «positive » des camps : décréter l'existence d'individus passibles d'internements et de détentions administratives, indépendamment des questions pénales, des responsabilités individuelles et des facteurs biographiques. L'empreinte que laissent les camps, leur «productivité » spécifique, réside donc dans le signe de la possibilité d'internement et de déportation. Dans l'absolue temporalité à laquelle ils condamnent, dans le provisoire qu'ils ratifient, les camps ne sont pas uniquement le seul territoire possible pour les individus déplacés, mais ils sont surtout ce qui, en raison de la menace même de leur existence, définit comme internable et déportable l'humanité qui excède les frontières, les territoires et les formes d'appartenance univoques. Les camps revêtent un caractère gouvernemental puisqu'ils constituent des lieux où le pouvoir est produit, des dispositifs à travers lesquels on institue une « différence » 18 .

On pourrait certes objecter à cela que toute forme de souveraineté est toujours dotée de dispositifs et d'instruments aptes à produire des différences, et que le pouvoir même (quelle qu'en soit sa forme, de la plus conventionnelle et la plus souveraine aux formes plus anonymes, microphysiques et disciplinaires d'une institution) s'est toujours incarné dans l'acte d'instituer une différence, aussi minime soit-elle. Sans nul doute, même dans l'exemple d'un camp, une certaine forme de souveraineté est toujours en jeu. Il s'agit cependant d'une souveraineté qui n'est pas seulement présupposée, mais qui simultanément se produit et produit; qui ne se contente pas de décréter des exceptions à son ordre interne, mais jaillit de (et agit sur) un espace radicalement différent par rapport aux frontières discrètes sur lequel le concept de souveraineté a trouvé son propre domaine d'application conventionnel. Dans la mesure où un tel espace excède toute dimension de frontière fondée sur un rapport dialectique entre un dedans et un dehors, et où il est progressivement investi sans jamais être absorbé et reconduit à l'intérieur de telles frontières, la forme de souveraineté qui se produit ainsi sera toujours quelque chose qui jaillira de (et agira sur) une série de sujets qui ne coïncideront plus avec les sujets nationaux.

18. La classification même d'exilé, de réfugié, de déplacé interne et de migrant (économique ou non) rentre à ce titre dans le cadre administratif de la gouvernementalité, comme la logique selon laquelle à chacune de ces typologies de sujets correspond un camp équipé déterminé. Il s'agit cependant d'une forme de gouvernementalité qui agit sur une «population » qui excède la population légitime et «souveraine » et, avec elle, l'espace administré de l'Etat-providence, travaillant sur un type d'extériorité pour lequel l'administratif impératif, «indifférent » aux biographies et à la vie, répond seulement à des critères « rationnels » : trier, importer, punir, déporter, interner, rapatrier. 
Ils ne se contenteront pas de faire exception aux formes de pouvoir étatiques et nationales, mais ils continueront à les excéder.

Nous aborderons de nouveau cette question par la suite. Pour le moment, nous nous contentons de suggérer l'idée que les camps dessinent une histoire parallèle, à part. Une histoire qui semble se placer dans cet espace « autre » que R.B.J. Walker définit comme l'" outside of the modern inside-outside 19 » (le "dehors du dedans-dehors moderne $20 »)$. Que cela soit bien clair, " histoire parallèle » ne signifie pas qu'elle n'entretient pas un rapport direct avec les espaces normés et les géographies politiques qui définissent l'appartenance. $\mathrm{Du}$ fait que les camps renforcent les frontières (géographiques et sociales), aujourd'hui toujours plus incertaines, et du fait qu'ils sont institués et administrés (ou de toute façon confirmés) par des Etats nationaux, il est simplement impossible de couper le fil qui les lie à l'extérieur, c'est-à-dire à cet « intérieur » à partir duquel ils se développent comme extériorité radicale. Il s'agit cependant d'un rapport qualitativement incommensurable par rapport à celui qui caractérise n'importe quel autre dispositif de détention qui agit à l'intérieur d'un territoire et d'un système juridique déterminés (prisons, asiles d'aliénés, hôpitaux psychiatriques - appelés « institutions totales » après les travaux, entre autres, de Michel Foucault et d'Erving Goffman) : un rapport qui n'est pas fusionnel, dans lequel toute «dialectique » entre exclusion et inclusion, entre un dedans et un dehors, disparaît.

\section{Excédent}

Cette distinction, entre autres, nous permet de retourner sur un point particulièrement controversé qui, depuis Arendt, caractérise une grande partie de la réflexion sur les camps d'internement : l'idée de l'inutilité qui semblerait unir les camps et l'humanité destinée à y trouver asile. L'hypothèse arendtienne trahit en effet une certaine idiosyncrasie envers tout ce qui ne se représente pas comme abstraitement politique, qui ne se trouve pas le long de la frontière absolue - pour Arendt une véritable finis terrae - qui sépare celui qui appartient de celui qui n’appartient pas, le citoyen de l'apatride. Mais ne pas appartenir ne signifie pas ne pas produire. Au contraire. Il n'est pas exact non plus que les camps (tous les camps, ceux d'hier et d'aujourd'hui) sont des lieux nécessairement improductifs, à perte. Cependant, leur productivité est une productivité sui generis qui ne répond pas à des logiques immédiatement économiques (exception faite des camps de travail forcé), ni de manière plus prosaïque à des logiques « fonctionnelles » (s'il est vrai, par exemple, que la grande majorité des immigrés détenus dans les centres de per-

19. Walker R.B.J., Inside/Outside: International Relations as Political Theory, Cambridge, Cambridge University Press, 1993 ; Walker R.B.J., «L'enigma dell'internazionale », Conflitti Globali, n², Milan, Shake Edizioni, 2005.

20. Kaminski A., I campi di concentramento dal 1896 a oggi. Storia, funzioni, tipologia, Torino, Bollati-Boringhieri, 1997 ; Kotek J., Rigoulot P., Le Siècle des camps. Détention, concentration, extermination, cent ans de mal absolu, Paris, J.-C. Lattès, 2000. 
manence temporaire est relâchée et non expulsée). Une productivité qui consiste justement à « donner forme » et à ratifier une différence radicale : en définissant celui qui est passible d'internement, puis en gérant les corps des internés - à savoir en les disciplinant, en les assujettissant, en les administrant, en les clandestinisant.

Productivité des camps, donc, qui répond à la condition particulière d'excédent de ceux qui y sont confinés, et aussi à la productivité spécifique qui caractérise un tel excédent. Certes, définir comme " productive » la condition de celui qui est contraint à s'exiler semble invraisemblable. Si l'on exclut les économies spécifiques que l'assistance humanitaire peut développer autour de tels sujets, l'intensité dramatique des déportations et des déplacements forcés semble au contraire nous restituer le sens désolé de quelque chose qui se trouve "endehors des calculs », qu'Arif Dirlik invite à développer de manière globale :

«Les “basket cases”, tous ceux qui n’ont rien à perdre et qui ne sont pas essentiels - les quatre cinquièmes de la population mondiale, en se basant sur les estimations fournies par les managers "mondialisés" n'ont pas besoin d'être colonisés : il suffit de les marginaliser. La nouvelle production flexible a rendu obsolète le recours à des formes explicitement coercitives et disciplinaires du travail vivant, aussi bien “chez nous” qu’à l'extérieur, dans les colonies. Les personnes et les lieux qui ne répondent pas aux nécessités du capital, ou qui sont désormais trop éloignés pour pouvoir y répondre efficacement, finissent tout simplement par se trouver en-dehors de ses calculs ${ }^{21}$. »

Ces propos nous font penser à une " mise à l'écart absolue ", à des années lumières de tout investissement « productif ». C'est ainsi, par exemple, qu'apparaît la condition d'une humanité en sureffectif qui envahit un bidonville ou un camp de réfugiés : individus exclus des logiques économiques, pour lesquels le langage courant de l'exploitation ne suffit plus. Pourtant, il semble que l'«inutilité » tragique restituée par les millions de «basket cases » que le présent produit, nous permette de lire l'excédent en termes exclusivement économiques, c'est-à-dire comme superflu parce qu'improductif. Du reste, la nouvelle production «flexible » a recours à des formes de coercition du travail vivant (travail domestique, travail des mineurs, travail forcé) qui envahissent aussi bien les colonies que les métropoles, qui voient se superposer et cohabiter maquiladoras et sweatshops ${ }^{22}$, travail immatériel et servile, sub-

21. Dirlik A., "The postcolonial aura", Critical Inquiry, automne 1994, p. 351.

22. «Ateliers de misère ", "sweatshops", "maquiladoras »... Chaque langue a ses mots pour désigner ce phénomène. S'il n'existe pas de définition légale, il caractérise ces manufactures dont la production est entièrement tournée vers l'exportation et se concentrant essentiellement sur les produits textiles, du cuir, du jouet, de l'équipement automobile ou encore sur les composants électroniques. Lorsqu'on parle des «ateliers de misère », ou sweatshops, on parle d'exploitation à outrance des travailleurs (mesures disciplinaires arbitraires, salaires au-dessous des normes minimales, insécurité au travail...). On parle aussi d'exploitation des enfants 
sumption formelle et réelle. Et les migrations contemporaines restituent une phénoménologie de l'excès qui répond à des logiques indubitablement productives, en général sous le signe de l'exploitation : une humanité exploitée parce que justement non représentée ni reconnue, parce qu'elle est clandestinisée, parce qu'en excès. Le fait que l'on fasse la distinction entre un excédent immédiatement productif et celui, en revanche, absolument « résiduel », abstraitement politique, même si cela rend la lecture immédiate plus facile, finit par reproduire et légitimer certaines divisions comme celles entre migrants, réfugiés et déplacés internes, perdant ainsi un continuum (même biographique) qui est le symbole primaire de l'excès contemporain. Il nous semble que le véritable sens de l'excès, en tant que condition au-delà de toute forme d'appartenance, se définit matériellement par un travail séparé de tout droit et une existence non représentée, rendant opaque toute distinction entre productivité et improductivité, exploitation et abandon. En d'autres termes, c'est autour de la fracture profonde qui s'insinue entre le travail et les droits et entre la vie et chaque contexte reconnu, que se construit la véritable frontière de l'excédent : une frontière politique qui est aussi une frontière économique ; une frontière qui désarticule et réarticule toute relation entre l'économique et le politique. Et de là émerge, comme fil rouge, une image agrandie et difforme du spectre quotidien de la précarité qui définit l'existence - pour reprendre l'expression de Castles et Davidson - aussi bien des non-personnes, les apatrides d'aujourd'hui, que des millions de « citadins qui n'appartiennent pas » ou dont l'appartenance est réduite à une ombre privée de toute substance.

C'est pour cette raison que l'excédent dépasse les frontières politiques et géographiques : il indique la présence de sujets qui, au-delà d'une exploitation immédiate et totalement coupée d'une biographie possible, peuvent être comptés et classifiés mais qui, de toute façon, ne comptent pas. Il fait allusion à l'existence, comme trait tout à fait décisif de ce présent, d'une part énorme d'humanité alternativement « utilisable et jetable ». Et l'excédent désigne les conditions de vie de ces existences en transit qui se réduisent à une simple reproduction biologique (celle qui, au nom des droits humains, est garantie dans un camp de réfugiés), et à la mort possible (d'une mort non recensée et anonyme, un « naufrage » sans spectateurs comme celui dont Kanafani nous fait le récit). C'est à cette humanité en excès que se présente la figure menaçante d'un camp d'internement, comme seul territoire possible, et qui ratifie une condition qui va au-delà de ce que l'on entend sociologiquement et politiquement par exclusion. Une condition qui matériellement indique la crise globale dans laquelle se précipite un système inclusif, une idée de droit, une forme d'appartenance. Les camps, en ce sens, signalent la manière dont se réarticule les nouvelles différences de statuts, de classe et de « race » dans le cadre des processus de déterritorialisation mondiaux. Ce sont des activateurs de la différence qui sanctionnent

et particulièrement des jeunes filles. On les retrouve essentiellement en Amérique Centrale et en Asie, mais aussi en Afrique et dans certains pays de l'ancienne Europe communiste. 
une condition qui excède toute représentation conventionnelle en termes d'exclusion et qui vient probablement «incluse » 23 (ou mieux recluse) sur des bases radicalement différentielles, voire d'un véritable apartheid ${ }^{24}$.

\section{Une généalogie}

Si les camps désignent une histoire à part, cette histoire naît loin de l'Occident. De nombreux travaux sur les camps (à partir de ceux que Hannah Arendt n'a pu malheureusement qu'ébaucher, en passant par les travaux «plein d'amertume » d'Andrej Kaminski, pour arriver aux écrits plus récents et discutables de Kotek et Rigoulot) ont mis en évidence les origines coloniales de cette histoire. Les premières expérimentations d'internement et de détention administrative de « civils » ont eu lieu, en effet, dans ce « laboratoire » particulier qu'ont été les colonies : à Cuba, par les Espagnols, en 1894 ; six ans plus tard en Afrique du Sud par les Anglais, ce qui a marqué la véritable inauguration $\mathrm{du}$ " siècle des camps 25 ». Ses origines pourraient se complexifier si nous remontions jusqu'aux réserves où furent confinés les peuples autochtones d'Amérique du Nord ; mais si, en revanche, on suit l'idée gramscienne de colonisation (avec toutes ses spécifications discursives, dont la plus centrale est celle d' « espace vital »), alors la matrice coloniale semble encore plus confirmée par ce précédent historique. Il subsiste cependant une lacune, partiellement imputable à Arendt, qui dénonce au contraire avec force les «massacres administratifs » conduits par les puissances coloniales, mais qui apparaît plus préjudiciable dans les travaux successifs : cette lacune concerne la capacité à s’interroger sur le sens de ces origines, sans se limiter à en prendre acte comme une simple donnée historique.

Il faut souligner que les camps ne sont pas le seul «instrument » né dans les colonies puis introduit en métropole par la suite, contrairement à la rhétorique dominante selon laquelle l'Histoire se serait toujours déroulée d'abord dans les métropoles puis dans les colonies. Dans un essai de 1979 26, Carlo Ginzburg retrace l'origine bengalaise des empreintes digitales, pratique à laquelle avait recours la population locale pour ranger les individus dans un système familial et de caste, qui par la suite a été adopté par le système colonial anglais comme dispositif d'identification et de contrôle : quand les empreintes franchiront la frontière absolue entre métropoles et colonies, elles iront soutenir une frontière interne

23. On peut voir à ce sujet Mezzadra S. (dir.), I confini della libertà, Rome, Derive Approdi, 2004.

24. Etienne Balibar, en particulier, dénonce la manière dont le processus d'intégration et de constitutionnalisation de l'espace européen prend le chemin d'un véritable régime d'apartheid, marqué par la présence d'une sous-catégorie de citoyenneté, la citoyenneté des migrants, dont Balibar rappelle le caractère post-colonial et la condition de "déportabilité ». Voir Balibar E., L'Europe, l'Amérique, la guerre. Réflexions sur la médiation européenne, Paris, La Découverte, 2003 ; Balibar E., "Europe, pays des frontières », Europe Constitution Frontière, Paris, Editions du Passant, 2005.

25. Kaminski A., op. cit. ; Kotek J., Rigoulot P., op. cit.

26. Voir Ginzburg C., Mythes, emblèmes, traces. Morphologie et histoire, Paris, Flammarion, 1989. 
fondamentale, celle entre les «classes laborieuses » et les «classes dangereuses », en permettant la transition, dans un certain sens, entre pratiques disciplinaires et formes de contrôle social. On assiste presque au même scénario, bien que plus dramatique, avec la mitrailleuse : après avoir donné la preuve de ses potentialités meurtrières lors de la guerre civile américaine, son utilisation fut interdite lors des guerres qui se déroulèrent en « Occident » pour finalement jouer un rôle décisif dans la course à la colonisation de l'Afrique et être employée sans trêve, toujours aux Etats-Unis, lors des dernières campagnes contre les autochtones et les répressions féroces des grèves des ouvriers de la fin du XIXe siècle. L'irruption des mitrailleuses dans les tranchées de la Première Guerre mondiale signifie alors qu'on a franchi le pas, et que la « guerre totale » pratiquée dans les colonies envahit désormais le cœur des métropoles. Ce mouvement de la périphérie vers le centre reste essentiel pour comprendre les événements «métropolitains ». Ainsi, Aimé Césaire, grand intellectuel martiniquais, activiste anticolonialiste et théoricien de la négritude, nous invitait à voir dans les fascismes européens l'importation, au cœur de l'Europe, de pratiques coloniales, après le partage à Berlin, en 1885, de l'« espace vital » des colonies ${ }^{27}$. Pour Cesaire, le véritable tabou brisé par le nazisme, l'horreur au-delà de l'horreur suscitée par Auschwitz, résidait (aussi) dans le fait, jusqu'alors impensable, de pouvoir utiliser directement sur les « citoyens » des métropoles ce qui avait été concevable uniquement pour les sujets des colonies, pour cette humanité en excès dont la vie et la mort s'avéraient des options absolument indifférentes et indifféremment praticables. Ce dépassement particulier, qui nous permet de situer l'Holocauste dans une dimension par certains côtés déjà " post-coloniale », nous ordonne surtout d'observer de plus près cette situation de violence absolue, «silencieuse et sans rédemption », qu'a été le colonialisme. Elle nous oblige en particulier à réfléchir sur une condition, celle de sujet, à laquelle il semble nécessaire de se confronter pour comprendre le sens de la forme-camp dans l'histoire de la modernité. Au fond, une généalogie des camps c'est aussi, obligatoirement, une généalogie de l'excédent, dont le sujet représente la matrice, mais également le point de non-retour 28.

27. Césaire A., Discours sur le colonialisme, 1955, Paris-Dakar, Présence africaine, 1989, p. 12 et suivantes. Le même discours, mais avec un ton encore plus radical, est tenu par Du Bois W.E., The Modern World and Africa, 1946, New York, International Publishers, 1992.

28. Dans un essai écrit il y a presque vingt ans désormais, dans lequel la figure générique du sujet était décomposée en fonction de la caste et du genre, Gayatry Spivak a tenté de montrer comment le statut « biopolitique » même du citoyen, de ce sujet progressivement investi et « assujetti » aux soins et au contrôle de la part de l'Etat moderne, se révèle pratiquement impensable si l'on ne tient pas compte des coûts matériels qu'une telle transition (une histoire que Foucault a écrit «à moitié ») a imposé aux colonies. Les droits, d'abord sociaux plus que politiques, se « paient » avec des droits dans une partie du monde qui ne doivent pas être niés dans l'autre partie du monde, à savoir par l'exploitation, l'esclavage, la mort « insacrifiable » (NdT : fait réfèrence au concept de «vie insacrifiable » dans l'œuvre de G. Agamben). Si les conquêtes des métropoles pouvaient être véritablement soustraites aux colonies, cela ressemblerait à un jeu à somme nulle. En réalité, ce qui agit dans l'univers colonial est une absence radicale de droits, et de cette absence, peuplée de sujets en excès, naissent les camps. Voir Spivak G.C., "Can the subaltern speak?", in Grossberg L., Nelson C., (dir.), Marxism and the Interpretation of Culture, Urbana, University of Illinois Press, 1988, pp. 271-313. 
Refaire le parcours des trajectoires irrégulières dessinées par la « formecamp ", à partir de ses origines coloniales, signifie alors se retrouver devant un mouvement «élastique ", une histoire qui, partant des colonies, est destinée à se focaliser sur l'Europe - «provincialisant » l'Europe même - pour ensuite se réaffirmer dans le scénario postcolonial actuel, problématique et illimité. Les camps d'internement pour civils seront en effet importés en "Occident » au moment de la Première Guerre mondiale, sous la forme spécifique de structures de détention pour prisonniers de guerre et de camps de travail, dans un premier temps, et de lieux où l'on interne des civils de nationalité « étrangère ", par la suite. Puis, l'Europe des années 1920 et 1930, saturée de frontières, est envahie par les camps, sous la forme d'un scénario encore colonial et frôlant la catastrophe; catastrophe qui trouve dans Auschwitz l'événement destiné à mettre un sceau définitif à ce siècle et à l'histoire de l'humanité. Après l'abysse des lagers et l'absolu des camps d'extermination, la présence de la « forme-camp » se dissipe sans toutefois disparaître, suivant l'affirmation des frontières bipolaires de l'après-guerre : elle se précipite dans l'enfer des goulags soviétiques et des camps de travail forcé en Chine et dans l'est de l'Europe ; et elle continue d'infester un monde entier en voie de décolonisation - en Algérie, en Indochine, au Kenya, en Angola. Le caractère spécifique du dispositif d'internement administratif et $\mathrm{du}$ «substitut aux patries perdues » ou jamais possédées, émerge de nouveau avec violence vers la fin des années 1980, se disséminant dans la superficie apparemment lisse du présent. Voici, dans ses grandes lignes, l'histoire en soi dessinée par les camps. Nous avons dédié le chapitre central d'une monographie à la tentative de refaire ce parcours de plus près ${ }^{29}$. Nous n'entendons pas nous étendre ultérieurement sur les étapes de ce mouvement, si ce n'est pour rappeler trois éléments de réflexion étroitement liés.

\section{Des colonies au monde}

La première question, méthodologique, concerne la direction spécifique que prend un parcours généalogique. On entend essentiellement par généalogie l'hypothèse foucaldienne d'une "histoire du présent ", un mouvement anachronique qui, remontant dans le temps, restitue le présent comme alourdi par sa propre ombre projetée sur le passé ${ }^{30}$. En effet, c'est seulement à cette condition, d'un présent qui se redéfinit à travers le passé - en compliquant sa trame -, qu'il est possible de récupérer l'« histoire à part » des camps. Une histoire disant que ce qui est arrivé dans les camps, dans n'importe quel lieu et à n'importe quel moment, n'est peut-être pas explicable dans l'absolu, mais tire sûrement son origine du fait qu'un jour il a été décidé que les camps pouvaient exister, et que des êtres humains pouvaient y être confinés, devenant ainsi quelque chose d'autre. Le fait que ce jour soit historiquement et géographi-

29. Rahola F., op. cit., pp. 61-113.

30. Voir Foucault M., « Nietzsche, la généalogie, l'histoire », Hommage à Jean Hyppolite, Paris, 1971. 
quement lié aux colonies, nous permet de reconnaître à travers la figure du sujet colonial le «premier » sujet « internable » et « déportable » mais, surtout, reconduit tout discours sur les camps à un ordre spatio-temporel spécifique et à la frontière spécifique sur laquelle cet ordre se fondait.

Edward Said, dans les pages finales de Culture et Impérialisme, probablement le livre le plus important qu'il ait jamais écrit, invitait à relire la totalité du projet colonial occidental sur la base d'un « principe de confinement » plus général qui en constituait en même temps la boussole et la matrice de fond ${ }^{31}$. L'effet, en trompe-l'œil, était celui d'un monde dichotomique, coupé en deux le long de la frontière «physique et épistémique » qui séparait les métropoles des colonies, sanctionnant la coexistence de sujets et de citoyens dans un temps unifié (celui homogène et vide de l'historicisme) et une géographie rigidement polarisée. Autrement dit, les colonies représentaient un espace en excès, qui échappait et remettait en question toute image dialectique des frontières nationales, toute logique dedans-dehors et qui, pour cela, était ratifié par un droit colonial, lequel nécessairement « excédait » le système juridique de chaque puissance coloniale (plus que lui faire exception). Et c'est justement à travers cette dimension d'excès (dont la traduction discursive plus immédiate confluait avec la rhétorique d'un « espace vital » investi mais distinct, jamais absorbé en totalité) qu'agissait le principe de confinement, l'artifice en vertu duquel l'espace colonial pouvait coexister séparément avec celui, expansif, des métropoles en un temps progressif et synchronique (à savoir « moderne »).

Que l'ordre spatio-temporel des colonies et la frontière sur laquelle elles se fondaient soit aujourd'hui définitivement dépassés est un argument qui ne cesse de susciter des polémiques. Sans nier la persistance de rapports d'exploitation et de domination directe, d'occupations et de protectorats militaires qui servent constamment de corollaire aux guerres préventives ou d'ingérence, il serait réducteur de représenter le présent en termes itératifs et simplement (néo-)coloniaux; ne serait-ce que pour les bouleversements radicaux qui, à partir des luttes pour l'indépendance, ont redessiné, même si ce n'est qu'en superficie, une géographie entière. C'est pour cela qu'à partir de ce domaine d'étude surpeuplé et souvent dangereusement banalisant qui se rapporte au «postcolonialisme », il serait particulièrement utile d'étudier le sens d'une transition qui a eu lieu mais qui n'est absolument pas résolue, rendant problématique le sens en apparence évident du préfixe « post » 32 . De manière très synthétique, il s'agit de voir dans le présent les traces encore vives d'un passé de domination et d'exploitation qui ne passe pas, sans cependant pouvoir les

31. Said E., Culture et Impérialisme, Paris, Fayard, coll. « Le monde diplomatique », 2000.

32. Voir Mellino M., La critica postcoloniale. Decolonizzazione, capitalismo e cosmopolitismo nei Postcolonial Studies, Roma, Meltemi, 2005 ; Mezzadra S., Rahola F., "The Postcolonial condition. A few notes on the quality of historical time in the global present", Postcolonial Text, $\mathrm{n}^{\circ} 13$, Toronto, 2004. 
reconduire de manière linéaire à cette géographie polarisée, à cette frontière absolue. Et c'est là que réside au fond le mouvement particulier «à rebours » qui impose une généalogie : où le passé pèse sur le présent et où le présent continue à compliquer et bouleverser la trame d'un passé. Cela signifie entre autres que cette frontière aujourd'hui se déplace partout virtuellement, au Nord comme au Sud, à l'Est comme à l'Ouest, et va séparer les centres métropolitains des banlieues, les gated communities (résidences de luxe) postcoloniales dorées des bidonvilles et des favelas. Cela souligne fondamentalement que le fantasme de cette frontière encore brisée se projette sur les sujets qui l'excèdent: hier c'étaient les sujets des colonies, aujourd'hui ce sont les migrants, les réfugiés, demandeurs d'asile, c'est-à-dire des individus au-delà de toute forme d'appartenance, qui demeurent perpétuellement « hors place » parce qu'ils continuent de dépasser, violer et transgresser le fantasme de cette frontière brisée ${ }^{33}$. Enfin, pour retourner au thème central de ces pages, c'est toujours le fantasme de cette frontière brisée qui fait en sorte que la formecamp se dissémine dans la superficie du présent, peuplant de camps aussi bien les anciennes métropoles que les anciennes colonies.

\section{Guerre et camps}

Il existe un second élément important sur lequel il est nécessaire de réfléchir. Il s'agit de la relation directe qui lie le recours aux camps à l'« état de guerre » : une guerre qui reste cependant non déclarée, tout au plus prolongée, non reconnue, tout au plus dissimulée et, dans tous les cas, radicalement asymétrique, parce qu'elle se trouve au-delà des frontières sur lesquelles elle peut s'organiser et se donner une forme. Dans la tentative de dessiner les contours d'une généalogie des camps, on se retrouve en effet continuellement à faire face aux histoires d'insurrections, de révoltes, de frontières violées. C'est en réponse à des luttes, ou simplement à la violation des frontières, qu'on a eu recours pour la première fois au camp, en référence à une logique qui radicalisait ces frontières violées : à Cuba, après une insurrection des colonisés, comme d'ailleurs en Afrique du Sud avec la guerre des Boers (sans impliquer ici une autre frontière interne, cette « ligne de la couleur » entre les boers et les autochtones

33. Mais, on peut voir aussi le spectre de cette même frontière à l'intérieur des espaces reconnus de la citoyenneté, comme le démontrent ces millions de « citoyens » postcoloniaux confinés dans des quartiers ethniques et des banlieues qui parfois explosent, dénonçant le vide qui se trouve derrière la figure universelle du citoyen. Le message qui émerge avec force des périphéries parisiennes en révolte à l'automne 2005, est justement celui de cette condition paradoxale qui, avec un ton méprisant et raciste, a été définie comme « racaille » : populace composée de « rien d'autre que de citoyens » (mais de « deuxième génération » ou d'origine coloniale), dont la présence privée de présent finit par vider complètement le caractère universaliste des droits affirmés en 1789 exemple concret de la manière dont l'universalisme abstrait contribue à cacher la reproduction d'inégalités réelles. C'est pour cette raison que la banlieue ne semble pas si éloignée d'un camp de détention et elle pourrait donc aussi faire partie de la «forme-camp ». Pour une critique « décentrée » du concept abstrait et universaliste de citoyenneté, on peut consulter Chatterjee P., The Politics of the Governed, Columbia University Press, New York 2004 
qui a été violée mais pas encore abolie aujourd'hui); peut-être aussi en Namibie, où les Hereros "concentrés et exterminés » par l'exercice colonial allemand, qui par leur simple existence excédaient les frontières du Lebensraum allemand; certainement au Vietnam, en Algérie, au Kenya, en Angola, etc. Mais on peut encore compléter la liste, en y ajoutant les masses des "sans patrie » peuplant l'Europe des années 1920 et 1930 destinée à se remplir de camps (les mouvements ouvriers organisés, les apatrides en fuite) qui ont exprimé plus ou moins directement et de manière différente un potentiel subversif similaire : en contestant un principe d'inclusion qui se fondait sur des frontières péremptoires de race, de nation, de classe ; ou bien, plus simplement, en traversant de telles frontières parce que fuyant d'autres frontières qui se radicalisaient ou des ghettos où ils avaient été contraints de rester depuis des siècles. Dans un cas comme dans l'autre, de par leur présence « impossible », ils ont révélé la crise irréversible d'un modèle inclusif fondé sur leur exclusion, provoquant une réaction qui radicalisera l'exclusion à un autre niveau, c'est-àdire recourant à un dispositif de détention dont les origines ne reconduisaient pas à l'intérieur des systèmes nationaux, mais à un espace, colonial, qui excédait le rayon d'action de ces systèmes. Les camps arrivent donc en Occident. Leur introduction, outre le fait de représenter la possibilité de la suspension du droit à l'intérieur des Etats de droit, est le symptôme d'une exclusion qui, comme cela a été le cas pour les sujets coloniaux, dépasse l'exclusion même, dans la mesure où elle fait allusion à un espace qui excède toute logique "dedans-dehors ", toute figure discrète de frontière : un espace où la vie et la mort représentent de simples alternatives biologiques et, où la paix et la guerre sont des options indifféremment praticables et absolument réversibles.

Par ailleurs, le recours aux armes chimiques et les premières exterminations planifiées (les Allemands en Namibie, le général Graziani en Libye, les Français à Sétif), les premières victimes civiles répertoriées comme étant «fortuites ", l'utilisation de la torture comme pratique utilisée pour les ennemis qui ne sont pas reconnus comme tels, sont des éléments dont il est possible de déduire la matrice coloniale des frontières asymétriques d'aujourd'hui - avec leur corollaire de tortures, d'internements, enemy combatants, "dommages collatéraux », phosphore et uranium appauvri - sans plus pouvoir cependant faire référence à une idée absolue de frontière. Cela signifie entre autres que des lieux comme Abou Ghraib ou Guantanamo n'évoquent pas seulement une forme extrême de prison (et donc une logique d'exception par rapport à un système juridique qui devrait être suspendu), mais surtout semblent entrer pleinement dans la catégorie de la forme-camp, c'est-à-dire dans un espace extraterritorial qui, en tant que tel, excède tout système et toute dimension discrète de frontière : la projection déformée et globale - une fois que la garantie de l'ordre a été abolie par le partage colonial - des nombreux centres spéciaux où étaient concentrés et torturés ces sujets particuliers en excès, ces sujets coloniaux. 


\section{L'espace des camps}

La dernière question concerne la «qualité » de cet espace, et donc la place " géographique » et politique des camps. Nous avons fait allusion au fait que, dans une logique d'exception, les camps seraient le dispositif à travers lequel une certaine forme de souveraineté réussirait à concevoir et comprendre l'extérieur dans son propre intérieur. Ainsi, par exemple, on comprend la manière dont les centres de détention pour migrants peuvent être reconduits à l'intérieur d'un système national déterminé, à travers la suspension de ce même système. Le fait est que, à côté de la question «formelle »- tout aussi importante - de la suspension du système, et donc des éléments d'extra-légalité ou d'alégalité même de l'institution des camps, il est nécessaire de se concentrer sur les effets matériels que produit le recours à un tel dispositif. Dans ce cas, il s'agit de la différence radicale qui s'exprime quand un individu devient « internable », et donc de l'institution «matérielle » d'un double régime juridique à travers les camps. Autrement dit, plus proche de l'idée foucaldienne de gouvernementalité, quand on parle de pouvoir, il faut garder à l'esprit que les effets sont souvent plus importants que les causes, dans la mesure où la souveraineté même, au lieu d'être présupposée, est conçue comme quelque chose qui se produit et qui, en se produisant, institue des différences. C'est pour cette raison qu'une lecture d'exception - qui tend toujours à préférer le pouvoir institutif des lieux aux effets de pouvoir qui se produisent sur les sujets finit par inscrire l'institution des camps à l'intérieur d'une histoire vouée à se reproduire évoluant en circuit fermé, répétant continuellement une logique de souveraineté inaltérée, indestructible, omnivore. Cette logique insiste et « rebondit » de façon continue à l'intérieur des frontières nationales définies, c'est-à-dire à l'intérieur de cette dialectique binaire « dedans-dehors » à l'intérieur de laquelle l'idée de souveraineté s'est développée. Par rapport à cette trame, les camps semblent en revanche indiquer une radicale extériorité : ce sont des lieux absolument déterritorialisés, comme peut l'être le container dont parle Kanafani, et comme le démontre a posteriori le mouvement qui voit aujourd'hui les camps s'éloigner toujours plus des frontières nationales et assumer une dimension extraterritoriale. On pense alors aux centres «préventifs » de détention et d'identification pour les migrants et les demandeurs d'asile qui «défendent » les frontières externes de l'Union européenne (en Ukraine, en Géorgie, en Libye, au Maroc), ou aux «trous noirs » extraterritoriaux où sont internées (sans même que l'état d'exception soit déclaré) les personnes suspectées d'être des «terroristes », ou encore aux camps « humanitaires » qui, emprisonnant de manière préventive in situ, nient la possibilité de faire une demande d'asile dans un pays tiers. Existe-t-il des frontières définies où il est possible de reconduire ces « formes » de camp ? Quel type de souveraineté les institue et peut s'y exercer ? La réponse se trouve-t-elle seulement à l'intérieur des frontières traditionnelles et dans l'état d'exception, ou faut-il plutôt regarder ailleurs, dans un espace qui excède de telles frontières? 
Tout au long de ces pages, on l'aura compris, nous proposons de regarder ailleurs. C'est la raison pour laquelle, sans exclure complètement l'interprétation faite des camps comme étant des émanations exclusives d'une forme de souveraineté juridico-politique décrétant l'état d'exception, nous avons voulu mettre en avant une explication alternative, différente, dans la mesure où elle remet surtout en question les frontières à l'intérieur desquelles les camps seraient institués et l'exclusion serait incluse. D'ailleurs, l'idée promue par Césaire selon laquelle les totalitarismes ont importé en Europe ce qui était considéré comme normal dans les colonies n'indique pas seulement la possibilité de voir une continuité à l'intérieur d'une histoire, mais plus radicalement la nécessité de décentrer cette histoire, de la lire à partir d'autres coordonnées. Certes, on pourrait toujours objecter que, dans les colonies justement, l'état d'exception était la règle. Mais il nous semble qu'il émerge des colonies en particulier, une «qualité » différente de l'espace politique et une forme différente de souveraineté qu'il ne faudrait pas se limiter à reconduire à la souveraineté stato-nationale à travers la catégorie passe-partout de l'« exception » 34 .

L'espace des colonies était en effet et avant tout un espace « autre », qui en tant que tel a toujours excédé et remis en question toute logique binaire (dedans-dehors, ami-ennemi) et toute représentation des frontières comme «simples » lignes de discontinuité entre territoires différemment qualifiés en termes de souveraineté. Un espace qui serait, caractérisé par une " guerre permanente ", de «basse intensité ", sans qu'il y ait nécessité de décréter des lois martiales ou l'état d'exception - nécessité qui faisait loi au point qu'il était impossible pour un système d'agir tout en se révoquant ${ }^{35}$. Au fond, le célèbre aphorisme de Clausewitz pour lequel la guerre serait « la continuation de la politique par d'autres moyens ", insiste vraiment, si on approfondit sa lecture, sur cette extériorité particulière et absolue, où la guerre encercle la politique et lui succède, en représente la continuation au-delà de ses frontières, de son domaine d'application, du lieu conventionnel dans lequel s'est exercée et consommée l'idée moderne de souveraineté 36 .

34. Comme le suggère Partha Chatterjee, dans les colonies, les technologies gouvernementales précèdent l'Etat-nation : dans un pays « ethnographique » comme l'Inde, les pratiques de gouvernement de la population (recensements, déplacements forcés, classifications) agissaient sur des sujets auxquels on ne reconnaissait aucune souveraineté. Cela signifie que lorsqu'on excède toute forme de reconnaissance, l'Etat et les institutions agissent seulement sur le côté technique, gouvernemental. Alors, la matrice coloniale de la forme-camp renvoie plus à l'événement microphysique, invisible et anonyme de la gouvernementalité qu’à l'événement « imposant » de la souveraineté. Il en constitue, si l'on veut, l'autre face, réservée à ceux qui excèdent toute reconnaissance et tout droit. Et l'élément menaçant de cette histoire, réside moins dans sa dimension «d'exception » que dans ses effets procéduralement punitifs, c'est-à-dire dans la frontière et dans les différences qui se produisent. Voir P. Chatterjee, op. cit., 2006.

35. Voir sur ce thème Young R.J., Postcolonialism: An Historical Introduction, Oxford, Blackwell, 2001, en particulier le chapitre "The subject of violence", pp. 293-335.

36. Voir dans Conflitti globali, n¹, 2005, les contributions de Dal Lago A., «La guerra mondo » et Guareschi M., « Ribaltare Clausewitz ». 
C'est pourquoi l'origine coloniale de la forme-camp nous propose une direction différente et plus «éloignée »; une direction à l'aide de laquelle il ne s'agirait pas seulement (et pas tant) de voir comment les camps peuvent être compris dans un système juridique national en lui faisant exception, mais surtout de comprendre comment ces camps sanctionnent la limite du rayon d'action de ce système en l'excédant, marquant de manière plus prosaïque une frontière, entre exclusion et inclusion, entre dedans et dehors, qui n'existe plus.

Prendre en considération jusqu'au bout la matrice coloniale de la formecamp, et donc écrire une généalogie des camps, signifie les remettre dans l'espace auquel ils «appartiennent », c'est-à-dire dans ce «dehors » qui excède toute représentation discrète des frontières modernes et en indique en même temps l'artificialité menaçante et la faiblesse intrinsèque : «the outside of the modern inside-outside " («le dehors du dedans-dehors moderne », comme dans la formule, qui résonne presque comme un slogan suggérée par R.B.J. Walker ${ }^{37}$.

Les camps, dans cette perspective, représentent le symptôme d'un espace externe qu'une certaine littérature définit comme «international » et qui, aujourd'hui, devrait plutôt être qualifié de « mondial » : un espace qui a toujours excédé l'espace discret des nations dont historiquement il a été investi, dévasté, colonisé, razzié et "racialisé », mais jamais complètement absorbé. Un espace qui, c'est le sens postcolonial du présent mondialisé, à chaque fois qu'il pénètre dans l'espace discret des Etats nationaux, en violant et en abolissant leurs frontières, bouleversant toute distinction entre interne et externe, produit des trous, des gouffres. Et dans chacun de ces trous, dans chacun de ces gouffres, s'installe un camp.

37. L'hypothèse de Walker appréhende l'espace international comme l'extériorité à partir de laquelle la modernité politique et les logiques binaires (dedans-dehors, ami-ennemi) sur lesquelles elle s'est construite, ont pu se légitimer et constamment franchir les frontières. Voir Walker R.B.J., op. cit., 2005. 